\title{
Surgical outcomes after multiple segmentectomy: a cohort study
}

\author{
Seshiru Nakazawa ${ }^{1}$, Kimihiro Shimizu ${ }^{2}$, Natsuko Kawatani ${ }^{1}$, Kai Obayashi ${ }^{1}$, Yoichi Ohtaki ${ }^{1}$, \\ Takayuki Kosaka ${ }^{3}$, Toshiki Yajima ${ }^{4}$, Ken Shirabe ${ }^{1}$
}

${ }^{1}$ Department of General Surgical Science, Gunma University Graduate School of Medicine, Maebashi, Japan; ${ }^{2}$ Division of General Thoracic Surgery, Department of Surgery, Shinshu University School of Medicine, Matsumoto, Japan; ${ }^{3}$ Department of Thoracic Surgery, National Hospital Organization Takasaki General Medical Center, Takasaki, Japan; ${ }^{4}$ Department of Innovative Cancer Immunotherapy, Gunma University Graduate School of Medicine, Maebashi, Japan

Contributions: (I) Conception and design: S Nakazawa, K Shimizu, T Yajima; (II) Administrative support: S Nakazawa, K Shirabe; (III) Provision of study materials or patients: S Nakazawa, K Shimizu, N Kawatani, K Obayashi, Y Ohtaki, T Yajima; (IV) Collection and assembly of data: S Nakazawa, K Shimizu, N Kawatani, K Obayashi, Y Ohtaki, T Kosaka, T Yajima; (V) Data analysis and interpretation: All authors; (VI) Manuscript writing: All authors; (VII) Final approval of manuscript: All authors.

Correspondence to: Toshiki Yajima, MD. Department of Innovative Cancer Immunotherapy, Gunma University Graduate School of Medicine, Maebashi 371-8511, Japan. Email: yajimato@gunma-u.ac.jp.

Background: Segmentectomy is now a common treatment option for both lung cancer and metastatic lung tumors with increasing data and evidence. However, data on multiple segmentectomy of different lobes are scarce. Our objective was to clarify the clinicopathological features of multiple segmentectomy.

Methods: We reviewed patients who underwent segmentectomy between January 2010 and December 2019 at Gunma University Hospital. Multiple segmentectomy was defined as segmentectomy of different lobes during the same operation, in contrast to single segmentectomy, which was defined as segmentectomy of a single lobe. Clinicopathologic, operative, and postoperative results were compared between multiple segmentectomy and single segmentectomy.

Results: There were 324 patients who underwent single segmentectomy and 11 patients (12 cases) who underwent multiple segmentectomy. Multiple segmentectomy was mostly performed for treatment of metastatic lesions rather than lung cancer. The median number of resected segments was 1 (range, 1-5) in the single segmentectomy group and 3 (range, 2-4) in the multiple segmentectomy group. The median number of resected lung lesions was 3.5 in the multiple segmentectomy group. Multiple segmentectomy was associated with longer operative time, more bleeding, and longer drainage period and postoperative stay than the single segmentectomy group. There were no significant differences in severe complications as well as 30 and 90-day mortality.

Conclusions: Multiple segmentectomy is a lung-preserving procedure that can be considered for patients with multiple lung lesions and has feasible postoperative outcomes.

Keywords: Segmentectomy; simultaneous; lung cancer; metastatic lung lesion

Submitted Sep 26, 2021. Accepted for publication Nov 30, 2021.

doi: $10.21037 /$ jtd-21-1545

View this article at: https://dx.doi.org/10.21037/jtd-21-1545

^ ORCID: 0000-0001-6218-9396. 


\section{Introduction}

Segmentectomy is now increasingly performed for lung cancer and metastatic lung tumors (1). Surgical and oncological data are being accumulated, and the feasibility of segmentectomy is being acknowledged (2-6). The noninferiority of segmentectomy compared to lobectomy for the treatment of selected patients with lung cancer has recently been confirmed by the JCOG0802/WJOG4607L trial (6). Segmentectomy has also become an option for the resection of metastatic lung tumors (7). Much literature has focused on segmentectomy of a single lobe (single segmentectomy), further classified into typical/atypical, simple/complex, or common/uncommon segmentectomy, according to the segmental plane shape or familiarity among surgeons $(1,4,5,8)$. Although confusing, the term typical/simple/common and atypical/complex/uncommon usually refers to the same segments. That is, right or left $\mathrm{S}^{6}$, left upper division, and left lingular segment resection are all typical/simple/common segmentectomies. However, there is a discrepancy regarding basal segmentectomy (right $\mathrm{S}^{7-10}$ and left $\mathrm{S}^{8-10}$ ), which is classified as a typical/common segmentectomy, yet a complex segmentectomy $(1,4,5,8)$.

In parallel with the generalization of segmentectomy, we have seen an increase in patients presenting with multiple ground-glass nodules or with oligometastatic lung lesions from other cancers. Accordingly, more complicated procedures such as multiple segmentectomy will be required for resection of multiple lung lesions affecting different lobes. So far, the literature on features and outcomes of multiple segmentectomy is extremely scarce. Herein, we aimed to clarify the clinicopathological features and surgical outcomes of patients who underwent multiple segmentectomy and aimed to provide data for a safer popularization.

We present the following article in accordance with the STROBE reporting checklist (available at https://jtd. amegroups.com/article/view/10.21037/jtd-21-1545/rc).

\section{Methods}

\section{Study population and variables}

We retrospectively analyzed patients who underwent segmentectomy between January 2010 and September 2019 at Gunma University. Multiple segmentectomy was defined as multiple segmental resections of different lobes in the same operation, in contrast to single segmentectomy which was defined as a single segmental resection of a single lobe. Patients who had additional wedge resection were also included in each group (i.e., single segmentectomy with wedge resection was classified into the single segmentectomy group; multiple segmentectomy with wedge resection into the multiple segmentectomy group). The number of cases in our institute during the study period determined the sample size. In effort to decrease bias, we included consecutive cases of the same period for both groups. We compared clinicopathological factors between patients who underwent single segmentectomy and multiple segmentectomy. The study was conducted in accordance with the Declaration of Helsinki (as revised in 2013). The study was approved by Institutional Review Board of Gunma University Hospital (No. HS2019-279) and individual consent for this retrospective analysis was waived. Charts were reviewed for age, sex, comorbidities, past medical history, smoking history, respiratory function, computed tomography (CT) images, number of lung lesions, pathological diagnosis, and surgical outcomes. These surgical outcomes included surgical approach (thoracotomy or thoracoscopic), operative time, bleeding, duration of chest tube placement, postoperative hospital stay, complications, and 30- and 90-day mortality. Complications were subdivided and classified using the Clavien-Dindo classification. Major complications were defined as grade IIIa or greater complications $(9,10)$. Pulmonary fistula was defined as air leak lasting for more than 7 days or requiring adhesion therapy. Late-onset pulmonary fistula was defined as re-emergence of an air leak that required re-drainage. Resected specimens were examined histopathologically, and lung cancer was classified according to the World Health Organization classification scheme.

\section{Selection criteria for sublobar resection}

Intentional sublobar resection was performed for patients considered to have non-invasive lung cancer, metastatic lung lesions, or lung lesions without a definite preoperative diagnosis. Non-invasive lung cancer was defined as a lung lesion $\leq 2.0 \mathrm{~cm}$ with a consolidation/tumor ratio of $\leq 0.5$ (11). Compromised sublobar resection was performed for patients who could not undergo standard lobectomy because of limited cardiopulmonary reserve, as proposed by the Japanese Association for Chest Surgery guidelines (12). Segmentectomy or wedge resection was chosen depending on the location and radiologic features of the lung nodule, that is, segmentectomy was performed for non-palpable 


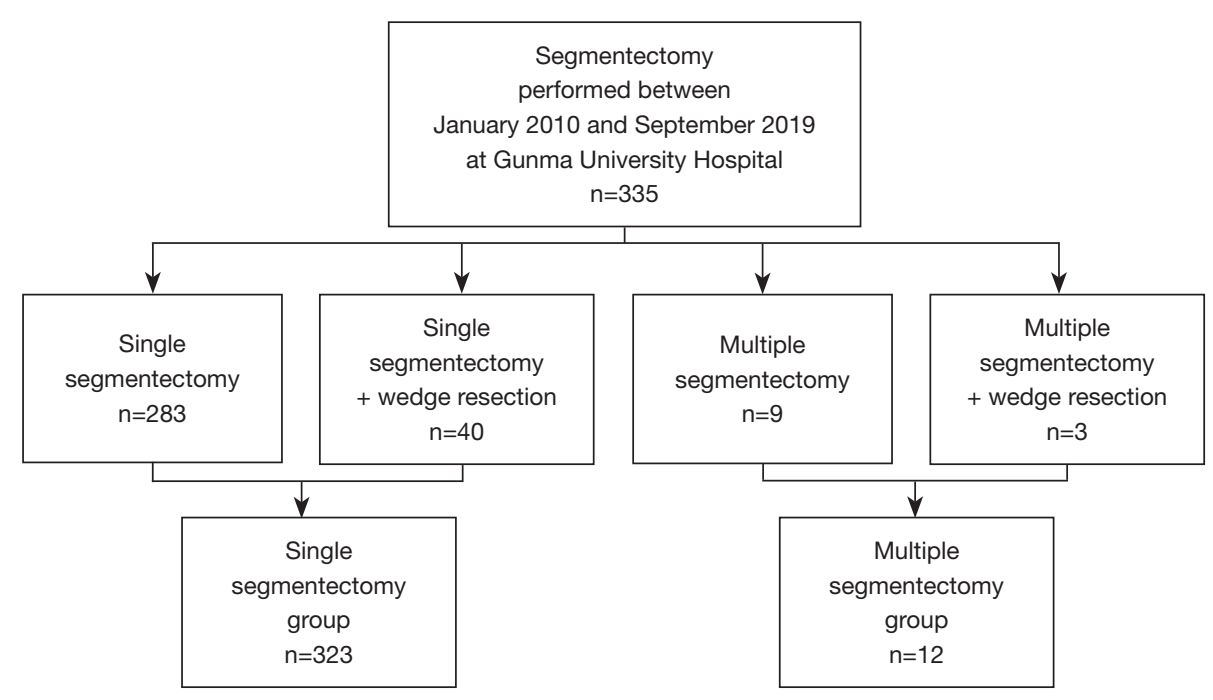

Figure 1 Flow diagram of patient selection.

nodules or for nodules located deep in the lung parenchyma or close to the hilum; wedge resection was performed for a palpable nodule for which sufficient surgical margin could be achieved.

\section{Surgical procedure of segmentectomy}

The basic technique for segmentectomy at our institute was previously described (1). Segmentectomy was planned according to the targeted segment. 3D-CT images, if available, were used for preoperative planning and intraoperative guidance (13). We used intersegmental veins to delineate the central part of the intersegmental plane. The peripheral part of the intersegmental plane was identified using the inflation-deflation line created by selective jet ventilation of the targeted segmental bronchus. After September 2018, we also used intravenous injection of indocyanine green after resection of the targeted pulmonary arteries to detect the intersegmental plane. The intersegmental plane was dissected by either electrocautery or stapling. Stapling was selected for patients with emphysematous lung or interstitial lung disease, or when the demarcation line of the intersegmental plane was unclear.

\section{Statistical analysis}

Summarized data are shown as median with interquartile range (IQR) for continuous variables, and as number and percentage for categorical variables. The Chi-square test was used to evaluate the relationship between categorical variables, whereas one-way analysis of variance, KruskalWallis test, and Mann-Whitney test were used for continuous variables. All reported $\mathrm{P}$ values were two-sided, with significance set at $<0.05$. Statistical analysis was performed using SPSS version 24 (IBM, Armonk, NY, USA).

\section{Results}

\section{Patient and tumor characteristics}

A total of 334 patients and 335 segmentectomies were included (Figure 1). There were also 16 cases of multiple lobectomy + segmentectomy cases during the same period, which were initially excluded as lobectomy cases. Among the 335 cases of segmentectomies, there were 323 cases of single segmentectomies (96\%) and 12 cases of multiple segmentectomies (4\%). Of the 12 cases of multiple segmentectomy, one patient had undergone multiple segmentectomy on both sides. Patient characteristics was analyzed based on 11 patients and surgical characteristics were based on the 12 cases. The number of segmentectomy cases increased, especially during the latter part of the study period (Figure S1). Among single segmentectomies, 153 were typical segmentectomies $(47 \%)$ and 170 were atypical segmentectomies (53\%). As detailed in Tables 1,2, there were no significant differences in age, sex, chronic obstructive pulmonary disease (COPD), pulmonary function, smoking, and laterality. The only difference was 
Table 1 Patient characteristics

\begin{tabular}{|c|c|c|c|}
\hline Characteristics & Single segmentectomy ( $n=323$ ) & Multiple segmentectomy $(n=11)$ & $P$ value \\
\hline Median (IQR) & $69(62-75)$ & $73(67-78)$ & \\
\hline Sex & & & 0.762 \\
\hline Women & $142(44 \%)$ & $4(36 \%)$ & \\
\hline \multicolumn{4}{|l|}{ Comorbidities } \\
\hline COPD & $35(11 \%)$ & $1(9 \%)$ & 1.00 \\
\hline Interstitial pneumonia & $5(1.5 \%)$ & $0(0 \%)$ & 1.00 \\
\hline Diabetes mellitus & $48(15 \%)$ & $3(27 \%)$ & 0.384 \\
\hline \multicolumn{4}{|l|}{ Pulmonary function } \\
\hline FEV1.0 (L) & & & 0.643 \\
\hline Median (IQR) & $2.32(1.78-2.90)$ & $2.27(1.87-2.77)$ & \\
\hline FEV1.0\% (\%) & & & 0.779 \\
\hline Median (IQR) & $76.7(70.8-82.5)$ & $75.3(69.4-84.7)$ & \\
\hline Smoking & & & 0.219 \\
\hline Yes & $185(57 \%)$ & $4(36 \%)$ & \\
\hline No & $138(43 \%)$ & $7(64 \%)$ & \\
\hline
\end{tabular}

IQR, interquartile range; COPD, chronic obstructive pulmonary disease; FEV, forced expiratory volume.

Table 2 Tumor characteristics

\begin{tabular}{lcc}
\hline Characteristics & Single segmentectomy $(\mathrm{n}=323)$ & Multiple segmentectomy $(\mathrm{n}=12)$ \\
\hline Laterality & $133(41 \%)$ & $5(42 \%)$ \\
Right & $190(59 \%)$ & $7(58 \%)$ \\
Left & & 3.00 \\
Pathological diagnosis & $198(61 \%)$ & $3(25 \%)$ \\
Lung cancer & $101(31 \%)$ & $9(75 \%)$ \\
Metastatic lung tumor & $23(7 \%)$ & 0.017 \\
Non-malignant & $1(0.3 \%)$ & $0(0 \%)$ \\
Others & & $2.0(1.1-3.3)$ \\
Preoperative tumor diameter $(\mathrm{mm})$ & $1.6(1.2-2.2)$ & 0.569 \\
Median (IQR) & & \\
\hline
\end{tabular}

$I Q R$, interquartile range. 

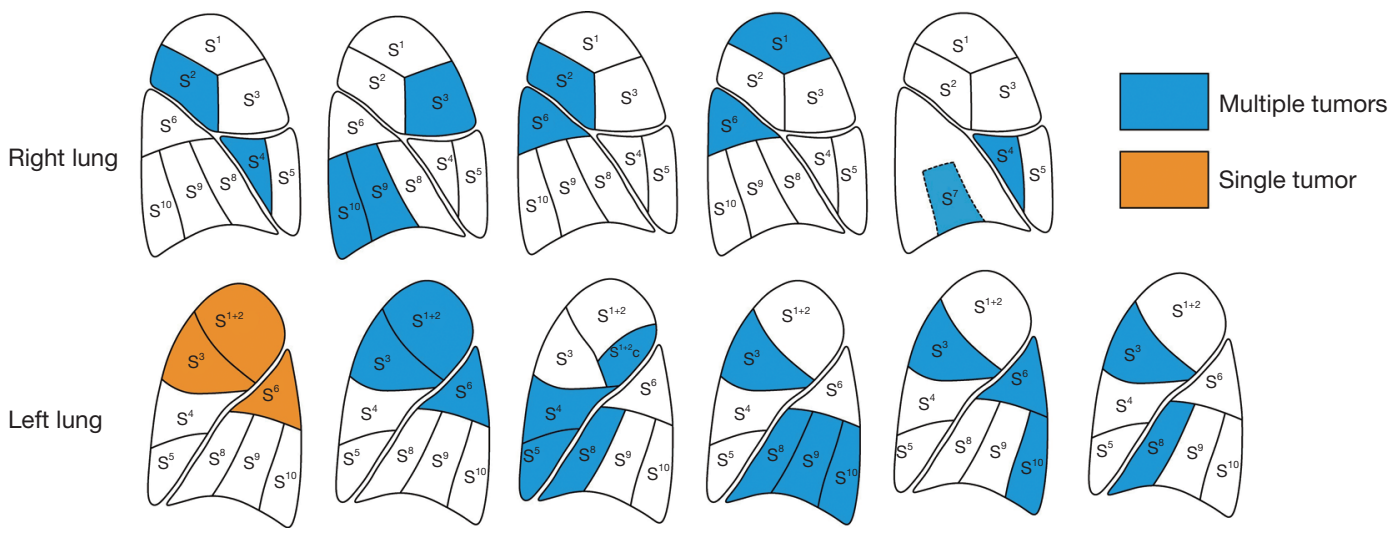

Figure 2 Schema of multiple segmentectomies. Multiple segmentectomies were performed either for resection of multiple lesions (blue) or for resection of a single lesion invading an adjacent lobe (orange).

Table 3 Details of multiple segmentectomy cases

\begin{tabular}{lcclcc}
\hline Segmentectomy \#1 & Segmentectomy \#2 & Additional resection & Laterality & Approach & $\begin{array}{c}\text { Number of resected } \\
\text { segments }\end{array}$ \\
\hline$S^{2}$ & $S^{4}$ & RLL wedge & Right & VATS & 2 \\
resected lesions
\end{tabular}

One patient underwent bilateral multiple segmentectomy. RLL, right lower lobe; VATS, video-assisted thoracoscopic surgery; IQR, interquartile range.

the pathological diagnosis of tumors. About $60 \%$ of patients undergoing single segmentectomy had the diagnosis of lung cancer, whereas only $25 \%$ of patients in the multiple segmentectomy group had lung cancer but rather a high ratio of metastatic lung tumor.

\section{Surgical features of multiple segmentectomy}

Figure 2 and Table 3 show an overview of segments resected in the multiple segmentectomy group. Most patients in the multiple segmentectomy group were treated for multiple lesions (10/12 cases, $83 \%)$ and metastatic lung tumors $(75 \%)$. However, two patients underwent multiple segmentectomy for resection of a single lesion invading the interlobar fissure (upper division $+\mathrm{S}^{6}$ segmentectomy). Of the 201 cases of lung cancer, 66\% ( $n=133)$ had lymph node evaluation of $\mathrm{N} 1$ station, $24 \%(\mathrm{n}=49)$ had evaluation up to N2 station, and $10 \%(\mathrm{n}=19)$ did not have lymph 

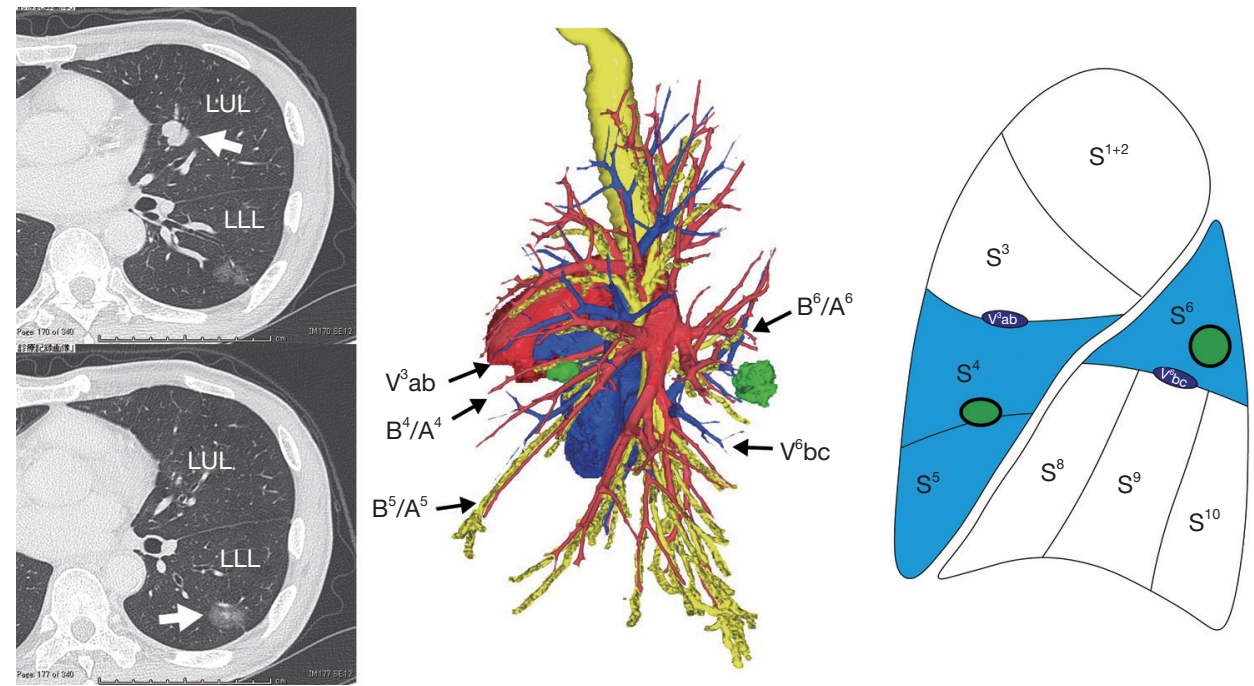

Figure 3 Images of a multiple segmentectomy case. Conventional CT images, 3D images, and schema of a multiple segmentectomy case (left lingula $+S^{6}$ segmentectomy) for the resection of a metastatic lung tumor from colon cancer (solid nodule in lingular segment) and a lung cancer (pure ground-glass nodule in $\mathrm{S}^{6}$ ). Pulmonary arteries are depicted in red, veins in blue, bronchi in yellow, and tumor in green. LUL, left upper lobe; LLL, left lower lobe; CT, computed tomography.

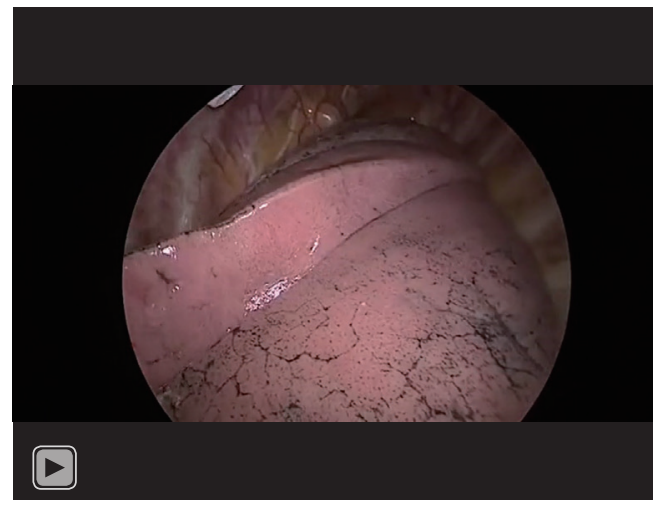

Video 1 Multiple segmentectomy case. Left lingula $+\mathrm{S}^{6}$ segmentectomy was performed for the resection of metastatic lung lesion and lung cancer.

node evaluation. The median number of dissected lymph nodes was 2 (IQR, 1-5) for the N1 station group, and 4 (IQR, 2-12) for the N2 station group. The most frequent type of multiple segmentectomies was a combination of atypical segmentectomies $(n=5,41 \%)$, followed by the combination of typical and atypical segmentectomies $(n=4$, $33 \%$ ), and finally a combination of typical segmentectomies $(\mathrm{n}=3,25 \%)$. Also, one patient underwent multiple atypical segmentectomies on both sides (right $\mathrm{S}^{4}+\mathrm{S}^{7}$ segmentectomy and left $\mathrm{S}^{3}+\mathrm{S}^{8}$ segmentectomy). A representative case of a recently performed multiple segmentectomy is shown in Figure 3 and Video 1.

\section{Surgical outcome and complications}

As shown in Table 4, patients in the multiple segmentectomy group, when compared to the single segmentectomy group, had a higher ratio of open approach ( $42 \% v s .8 \%)$, longer operative time (300 vs. $210 \mathrm{~min}$ ), and more blood loss (91 vs. $20 \mathrm{~mL}$ ), as well as longer length of drainage (median: $4 v s .2$ days) and postoperative hospital stay (median: $9 v s$. 6 days). There were 2 cases of conversion to thoracotomy in the single segmentectomy group (one due to bleeding and one for lymph node adhesion) and 1 case of conversion in the multiple segmentectomy group (due to severe adhesion). The conversion rate to thoracotomy was $0.7 \%$ (2 out of 299 cases) in the single segmentectomy group and $13 \%$ ( 1 out of 8 cases) in the multiple segmentectomy group. Postoperative complications are summarized in Tables 4,5. There were no significant differences in terms of overall complications, major complications, and in 30- or 90-day mortality. We experienced 1 case of postoperative bleeding after left upper division $+\mathrm{S}^{6}$ segmentectomy, for which the patient was treated conservatively. There were 3 cases $(0.9 \%)$ of re-operation for late-onset pulmonary fistula in the single segmentectomy group but no cases of re-operation 
Table 4 Perioperative characteristics

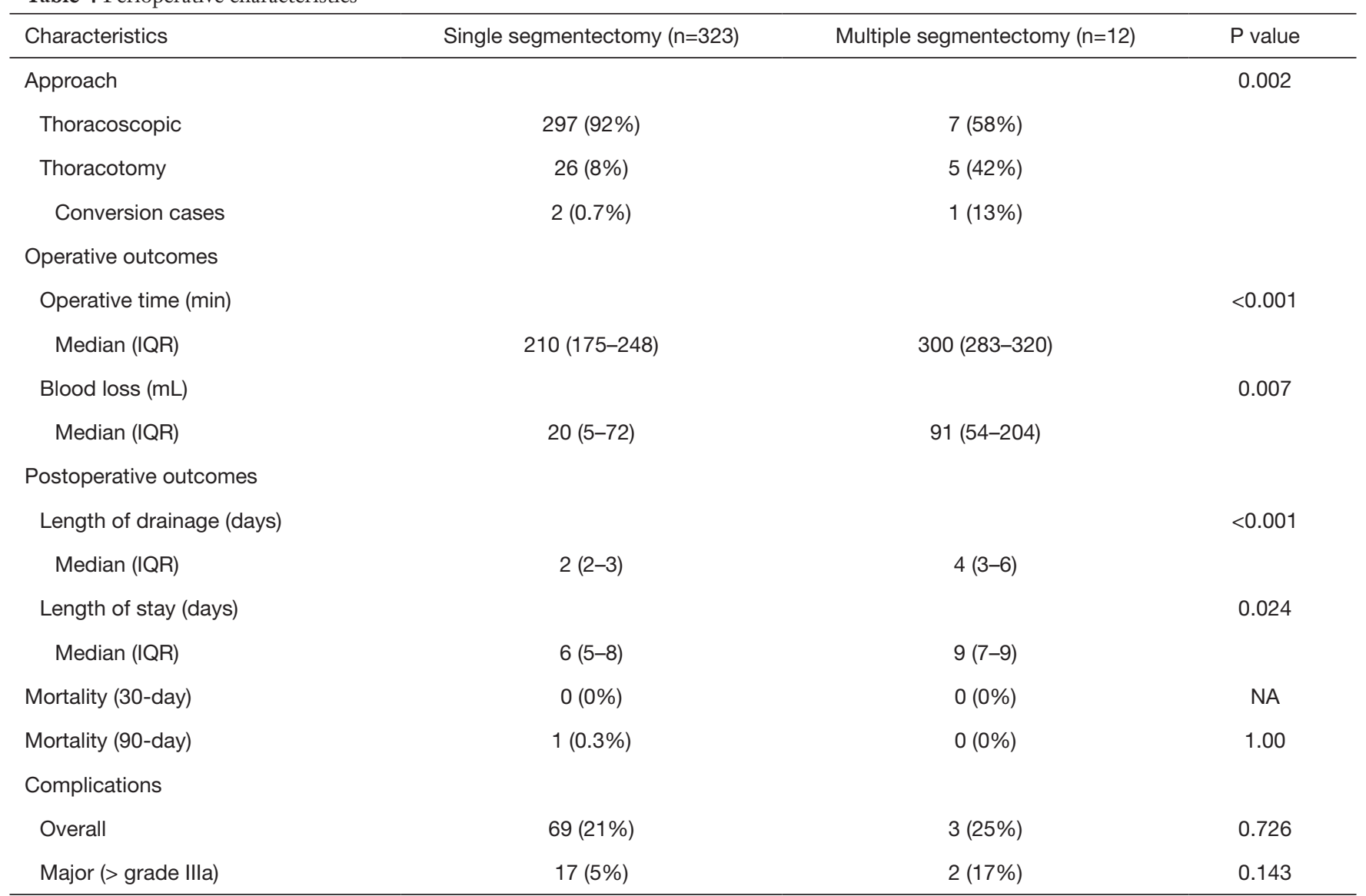

IQR, interquartile range; NA, not applicable.

in the multiple segmentectomy group. Also, no patient initially scheduled for multiple segmentectomy required intraoperative conversion to lobectomy due to oncological or surgical reasons, such as insufficient surgical margin or complications. We achieved complete resection for all cases in the multiple segmentectomy group, and there were 5 cases of incomplete resection in the single segmentectomy group ( 3 patients who underwent resection of lung cancer and 2 patients for resection of metastatic lung lesion).

\section{Discussion}

In the present study, we clarified the clinicopathological features and surgical outcomes of patients who underwent multiple segmentectomy in comparison to single segmentectomy. The key findings are as follows. Firstly, although patients who underwent multiple segmentectomy had a higher rate of thoracotomy, longer operative time, more blood loss, and longer drainage and hospital stay, there were no significant differences in major complications and mortality. Secondly, multiple segmentectomy was frequently chosen for treatment of metastatic lung tumors, rather than lung cancer. Thirdly, the most frequent type of multiple segmentectomy was a combination of atypical segmentectomies of different lobes.

The trend in perioperative outcomes of multiple segmentectomies were partly similar to those of previously reported atypical or complex segmentectomies, that is, longer operative time but similar rates in overall complications or mortality compared to their counterpart. For example, in an analysis of 128 atypical segmentectomies, operative time was longer, but there was no difference in drainage duration, postoperative hospital stay, and postoperative complications compared to typical segmentectomies (4). Similarly, another study on 117 complex segmentectomies also reported longer operative 
Table 5 Details of complications

\begin{tabular}{lcc}
\hline Characteristics & Single segmentectomy $(\mathrm{n}=323)$ & Multiple segmentectomy $(\mathrm{n}=12)$ \\
\hline Pulmonary complications & $22(7 \%)$ & $2(17 \%)$ \\
Pulmonary fistula & $17(5 \%)$ & $0(0 \%)$ \\
Late-onset pulmonary fistula & $9(2.8 \%)$ & $0(0 \%)$ \\
Pneumonia & $0(0 \%)$ & $0(0 \%)$ \\
Chylothorax & $0(0 \%)$ & $0(0 \%)$ \\
Empyema & $0(0 \%)$ & $0(0 \%)$ \\
Acute exacerbation of IP & & 1.000 \\
Other types of complications & $1(0.3 \%)$ & $\mathrm{NA}$ \\
Brain infarction & $0(0 \%)$ & $0(0 \%)$ \\
Recurrent nerve paralysis & $2(0.6 \%)$ & $0(0 \%)$ \\
Elevated liver enzymes & $0(0 \%)$ & $0(0 \%)$ \\
Arrhythmia & $0(0 \%)$ & $0(0 \%)$ \\
Postoperative bleeding & & 1.000 \\
NA & 1.000 \\
$\mathrm{NA}$
\end{tabular}

IP, interstitial pneumonia; N/A, not applicable.

time but no differences in 30-day mortality and overall complications compared to simple segmentectomies (5). Interestingly, some studies on complex or uncommon segmentectomies even showed no difference in operative time either. A study on 111 complex segmentectomies showed similar rates of 30-day mortality, morbidity, drainage length, and overall complications, but also no difference in operative time compared to simple segmentectomies (14). Furthermore, a study on 23 cases of uniportal uncommon segmentectomies also showed no differences in operation time, drainage length, hospitalization period, and postoperative complications compared with common segmentectomies (8). On the contrary, a post-hoc analysis of the segmentectomy arm in the JCOG0802/WJOG4607L study reported that complex segmentectomy was related to higher incidence of early postoperative complications (6). This randomized trial comparing segmentectomy and lobectomy included 300 cases of complex segmentectomies and 229 cases of simple segmentectomies. In our study, the higher blood loss, longer drainage period, and longer hospitalization in the multiple segmentectomy group might also be related to the higher rate of thoracotomy.

Regarding the surgical time, our results might be longer than other institutes for several reasons. Firstly, we use electrocautery for dissecting the intersegmental plane in about two-thirds of cases (one-third electrocautery alone, one-third electrocautery and stapler combined, and one third by stapler only, data not shown). This takes more time for both dissection and hemostasis. However, we believe that use of electrocautery is useful to achieve better expansion of the remaining lung. Secondly, we currently combine both selective jet ventilation and ICG injection to achieve better recognition of the intersegmental plane. This also takes more time for cannulation of the bronchus and marking of the lung surface.

Several limitations of this study should be considered. Firstly, this was a retrospective analysis with a small sample size for the multiple segmentectomy group with possible selection bias in surgical candidates and procedures. In effort to reduce bias, we included all consecutive cases of segmentectomy performed during the study period and compared multiple segmentectomy with single segmentectomy. More large-scale studies focusing on multiple segmentectomy are necessary to further clarify the features and feasibility of this procedure. Secondly, we analyzed only short-term outcomes; the long-term outcomes of multiple segmentectomy remains unclear. From a long-term oncological viewpoint, lung cancer cases lack data regarding proportion of ground-glass opacity in cases performed prior to the 8th TNM classification. 


\section{Conclusions}

In conclusion, multiple segmentectomy is a procedure that allows for the resection of multiple tumors of different ipsilateral lobes or of a single tumor invading more than two lobes. As expected, multiple segmentectomy is a procedure with longer operative time and higher blood loss, and it is also associated with longer drainage periods and hospitalization. However, it is of note that the rates of major complications and mortality did not differ compared to those with single segmentectomies. So far, the literature on multiple segmentectomy is extremely scarce. We predict that in parallel with the great increase of patients undergoing segmentectomy the need for simultaneous segmentectomies will also grow in the near future. We conclude that multiple segmentectomy is a feasible lungpreserving procedure that will benefit adequately selected patients.

\section{Acknowledgments}

We would like to thank Ms. Harumi Kanai for secretarial assistance, as well as Enago (https://www.enago.jp) for the English review.

Funding: This work was partly supported by KAKENHI Grant-in-Aid for Young Scientists Japan Society for the Promotion of Science (grant No. 19K18203 to SN).

\section{Footnote}

Reporting Checklist: The authors have completed the STROBE reporting checklist. Available at https://jtd. amegroups.com/article/view/10.21037/jtd-21-1545/rc

Data Sharing Statement: Available at https://jtd.amegroups. com/article/view/10.21037/jtd-21-1545/dss

Conflicts of Interest: All authors have completed the ICMJE uniform disclosure form (available at https://jtd.amegroups. com/article/view/10.21037/jtd-21-1545/coif). KS serves as an unpaid editorial board member of Journal of Thoracic Disease. The other authors have no conflicts of interest to declare.

Ethical Statement: The authors are accountable for all aspects of the work in ensuring that questions related to the accuracy or integrity of any part of the work are appropriately investigated and resolved. The study was conducted in accordance with the Declaration of Helsinki (as revised in 2013). The study was approved by Institutional Review Board of Gunma University Hospital (No. HS2019279) and individual consent for this retrospective analysis was waived.

Open Access Statement: This is an Open Access article distributed in accordance with the Creative Commons Attribution-NonCommercial-NoDerivs 4.0 International License (CC BY-NC-ND 4.0), which permits the noncommercial replication and distribution of the article with the strict proviso that no changes or edits are made and the original work is properly cited (including links to both the formal publication through the relevant DOI and the license). See: https://creativecommons.org/licenses/by-nc-nd/4.0/.

\section{References}

1. Nakazawa S, Shimizu K, Mogi A, et al. VATS segmentectomy: past, present, and future. Gen Thorac Cardiovasc Surg 2018;66:81-90.

2. Hwang Y, Kang CH, Kim HS, et al. Comparison of thoracoscopic segmentectomy and thoracoscopic lobectomy on the patients with non-small cell lung cancer: a propensity score matching study. Eur J Cardiothorac Surg 2015;48:273-8.

3. Stamatis G, Leschber G, Schwarz B, et al. Perioperative course and quality of life in a prospective randomized multicenter phase III trial, comparing standard lobectomy versus anatomical segmentectomy in patients with nonsmall cell lung cancer up to $2 \mathrm{~cm}$, stage IA (7th edition of TNM staging system). Lung Cancer 2019;138:19-26.

4. Xie B, Sun X, Qin Y, et al. Short-term outcomes of typical versus atypical lung segmentectomy by minimally invasive surgeries. Thorac Cancer 2019;10:1812-8.

5. Handa Y, Tsutani Y, Mimae T, et al. Surgical Outcomes of Complex Versus Simple Segmentectomy for Stage I Non-Small Cell Lung Cancer. Ann Thorac Surg 2019;107:1032-9.

6. Suzuki K, Saji H, Aokage K, et al. Comparison of pulmonary segmentectomy and lobectomy: Safety results of a randomized trial. J Thorac Cardiovasc Surg 2019;158:895-907.

7. Shiono S, Okumura T, Boku N, et al. Outcomes of segmentectomy and wedge resection for pulmonary metastases from colorectal cancer. Eur J Cardiothorac Surg 2017;51:504-10.

8. Matsuura N, Igai H, Ohsawa F, et al. Safety and feasibility 
of uniportal video-assisted thoracoscopic uncommon segmentectomy. J Thorac Dis 2021;13:3001-9.

9. Clavien PA, Barkun J, de Oliveira ML, et al. The ClavienDindo classification of surgical complications: five-year experience. Ann Surg 2009;250:187-96.

10. Dindo D, Demartines N, Clavien PA. Classification of surgical complications: a new proposal with evaluation in a cohort of 6336 patients and results of a survey. Ann Surg 2004;240:205-13.

11. Suzuki K, Koike T, Asakawa T, et al. A prospective radiological study of thin-section computed tomography to predict pathological noninvasiveness in peripheral clinical IA lung cancer (Japan Clinical Oncology Group 0201). J

Cite this article as: Nakazawa S, Shimizu K, Kawatani N, Obayashi K, Ohtaki Y, Kosaka T, Yajima T, Shirabe K. Surgical outcomes after multiple segmentectomy: a cohort study. J Thorac Dis 2022;14(1):113-122. doi: 10.21037/jtd-21-1545
Thorac Oncol 2011;6:751-6.

12. Sawabata N, Nagayasu T, Kadota Y, et al. Risk assessment of lung resection for lung cancer according to pulmonary function: republication of systematic review and proposals by guideline committee of the Japanese association for chest surgery 2014. Gen Thorac Cardiovasc Surg 2015;63:14-21.

13. Shimizu K, Nakazawa S, Nagashima T, et al. 3D-CT anatomy for VATS segmentectomy. J Vis Surg 2017;3:88.

14. Bédat B, Abdelnour-Berchtold E, Krueger T, et al. Impact of complex segmentectomies by video-assisted thoracic surgery on peri-operative outcomes. J Thorac Dis 2019;11:4109-18. 


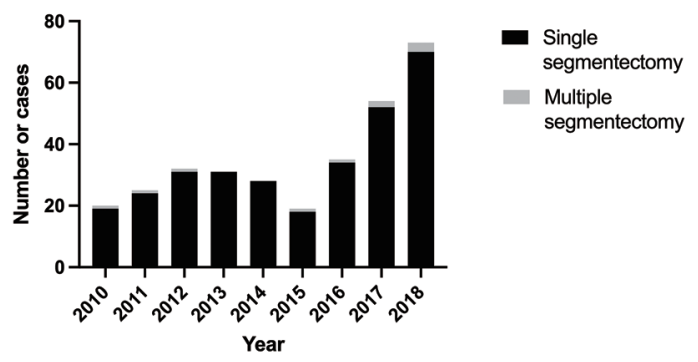

Figure S1 Change in number of segmentectomy cases during the study period. Single segmentectomies are shown in black and multiple segmentectomies in grey. Data from 2019 were omitted because study period was shorter than other years. 